\title{
Self-adaptive image denoising based on bidimensional empirical mode decomposition (BEMD)
}

\author{
Guo Song $^{\text {a, }}$, Luan Fangjun ${ }^{\mathrm{a}}$, Song Xiaoyu ${ }^{\mathrm{a}}$ and Li Changyou ${ }^{\mathrm{b}}$ \\ ${ }^{a}$ Information \& Control Engineering Faculty, Shenyang Jianzhu University, Shenyang, China \\ ${ }^{b}$ School of Mechanical Engineering and Automation, Northeastern University, Shenyang, China
}

\begin{abstract}
To better analyze images with the Gaussian white noise, it is necessary to remove the noise before image processing. In this paper, we propose a self-adaptive image denoising method based on bidimensional empirical mode decomposition (BEMD). Firstly, normal probability plot confirms that 2D-IMF of Gaussian white noise images decomposed by BEMD follow the normal distribution. Secondly, energy estimation equation of the $i$ th $2 \mathrm{D}-\mathrm{IMF}(i=2,3,4, \ldots .$.$) is proposed$ referencing that of $i$ th $\operatorname{IMF}(i=2,3,4, \ldots \ldots$ ) obtained by empirical mode decomposition (EMD). Thirdly, the self-adaptive threshold of each 2D-IMF is calculated. Eventually, the algorithm of the self-adaptive image denoising method based on BEMD is described. From the practical perspective, this is applied for denoising of the magnetic resonance images (MRI) of the brain. And the results show it has a better denoising performance compared with other methods.
\end{abstract}

Keywords: Image denoising, BEMD, self-adaption, Gaussian white noise, energy

\section{Introduction}

The existence of noise when obtaining or transmitting images makes it difficult for subsequent analysis. Image denoising is a low-level image processing to remove the noise involved when obtaining or transmitting images. It is an important front-end procedure for high-level visual tasks, including object recognition (e.g., magnetic resonance image analysis), digital entertainment, and remote sensing imaging, as it facilitate subsequent analysis [1]. Image denoising aims at removing noise as much as possible while maintaining the useful information to the largest extent. For a certain image, most of its energy lies in the low frequency band, while the details lie in the high frequency band. However, the noise energy distribution exists in the whole frequency domain. Generally, algorithms based on wavelet analysis are employed for image denoising [2-5]. Although it could simultaneously remove the noise while maintain the high frequency details of an image, yet it is difficult to choose the optimal wavelet mother function.

\footnotetext{
${ }^{*}$ Corresponding author: Guo Song, Information \& Control Engineering Faculty, Shenyang Jianzhu University, No. 9, Hunnan East Road, Hunnan New District, Shenyang City, Liaoning, China. Tel.: +86 24 24690666; Fax: +86 24 24690666; Email: guosong@sjzu.edu.cn.
}

0959-2989/14/\$27.50 @ 2014 - IOS Press and the authors. 
To analyze non-ideal and non-linear signals, Hilbert-Huang transform was proposed by Huang et al. in 1998 [6]. Hilbert-Huang transform consists of empirical mode decomposition (EMD) and Hilbert spectral analysis. The signal is firstly decomposed into the sum of several intrinsic mode functions (IMF) and the residue by EMD, then the instantaneous frequency and Hilbert spectrum are obtained [6,7]. In the transform, EMD essentially acts as a dyadic filter bank resembling involved in wavelet decompositions $[8,9]$. Therefore, the noise of the image or the signal might be removed by EMD. There exist several denoising methods based on the dyadic filter bank characteristic of EMD and they are mainly applied in the signal denoising [10,11]. EMD can also be combined with Savitzky-Golay filter, which can then be used to remove the drift noising of a dynamically tuned gyroscope [12]. Besides, EMD was also applied to image denoising by $\mathrm{Hu}$ and Jin who used the Savitzky-Golay filter to process the low-order IMFs while maintaining the high-order ones [13]. In addition, in electrostatic detection signal processing, people generally neglect the low-order IMFs and denoise the high-order ones using wavelet analysis [14]. Agarwal and Tsoukalas also proposed a denoising method based on the noise energy of IMFs [15] where IMFs with energy less than corresponding threshold would be removed. The Iterative EMD Interval-Thresholding (EMD-IIT) and Clear Iterative EMD IntervalThresholding (EMD-CIIT) denoising method were presented by Kopsinis and McLaughlin [16]. Subsequently, a self-adaptive threshold image denoising method based on their method was proposed by Guo et al. [17]. In this method, an image was firstly translated into a sequence and decomposed by EMD. Afterwards, the self-adaptive method was employed to remove the noise of each IMF. Due to the fact that the image might be distorted after being translated into the sequence, bidimensional empirical mode decomposition (BEMD) is used to decompose the noise image into 2D-IMFs and the self-adaptive method to be applied to each 2D-IMF to remove Gaussian white noise as much as possible.

\section{Self-adaptive image denoising based on BEMD}

\section{1. $B E M D$}

EMD has been reported to be used to remove the noise from images, such as in [13] and [17]. It has been also used to analyze images in other domains, such as in [18]. In those papers, images were firstly translated into one-dimensional sequences before EMD is applied. However, as those methods only carried out a one-dimensional EMD (not a true 2D-EMD), their limitation was found in local image analysis. Therefore, BEMD is used instead and it can be described as follows $[19,20]$ :

1. Initialization: $I_{0}=I_{\text {ori }}, m=1$;

2. Computation of the $m$ th $2 \mathrm{D}-\mathrm{IMF}$

1). Initialization: $H_{0}=I_{m-1}, j=1$;

2). Obtain all extrema of $H_{j-1}$;

3). Interpolate between extrema;

4). Compute the envelope mean (EM);

5). $H_{j}=H_{j-1}-\mathrm{EM}$;

6). If the stopping criterion is fulfilled by $H_{j}$, then $2 \mathrm{D}-\mathrm{IMF}_{m}=H_{j}$ and go to step 3 ; else $j=j+1$ and go to step 2);

3. $I_{m}=I_{m-1}-2 \mathrm{DIMF}_{m}$

4. If the number of extrema of $I_{m}$ is more than $2, m=m+1$ and repeat steps 2 and 3; else the decomposition is over. 
According to the Cauchy-type convergence condition, the stopping criterion is given by

$$
D=\sum_{x=1}^{M} \sum_{y=1}^{N} \frac{\left|H_{j}(x, y)-H_{j-1}(x, y)\right|^{2}}{\left|H_{j-1}(x, y)\right|^{2}} \leq 0.2
$$

The neighboring window method and the morphological reconstruction were employed to find local maxima/minima points and the extreme plateaus, respectively [19-21].

\subsection{Self-adaptive image denoising based on BEMD}

Image denoising method based on BEMD has been used by some researchers. For example, Pei et al. used the adaptive median filtering to remove the noise of each 2D-IMF obtained by BEMD [22]. In this work, the threshold was computed using the formula $\zeta \sigma \sqrt{2 \ln (N)}$, where $\varsigma$ was the modifying factor and it was generally the value of $0.25 . N$ was an arbitrary positive constant. Sharif also used BEMD in denoising aurora images [23], where Gaussian low pass filters were applied to the first few BIMFs after obtaining the 2D-IMFs. The filter width and shape could be varied from one 2D-IMF to the next. However, for users who are not expert in the images for denoising, it is difficult to determine the values of parameters such as $N$ in [22] and the filter width and shape in [23]. Therefore, the selfadaptive image denoising based on BEMD could be very useful.

The normal probability plot of each 2D-IMF of the Gaussian white noise image decomposed by BEMD is shown in Figure 1. Here, the pixels of the original image follow the standard normal distribution. It could be seen that the actual probability curve of each 2D-IMF almost coincides with the ideal one and the data range (horizontal ordinate of each sub-graph) reduces with the increase of the
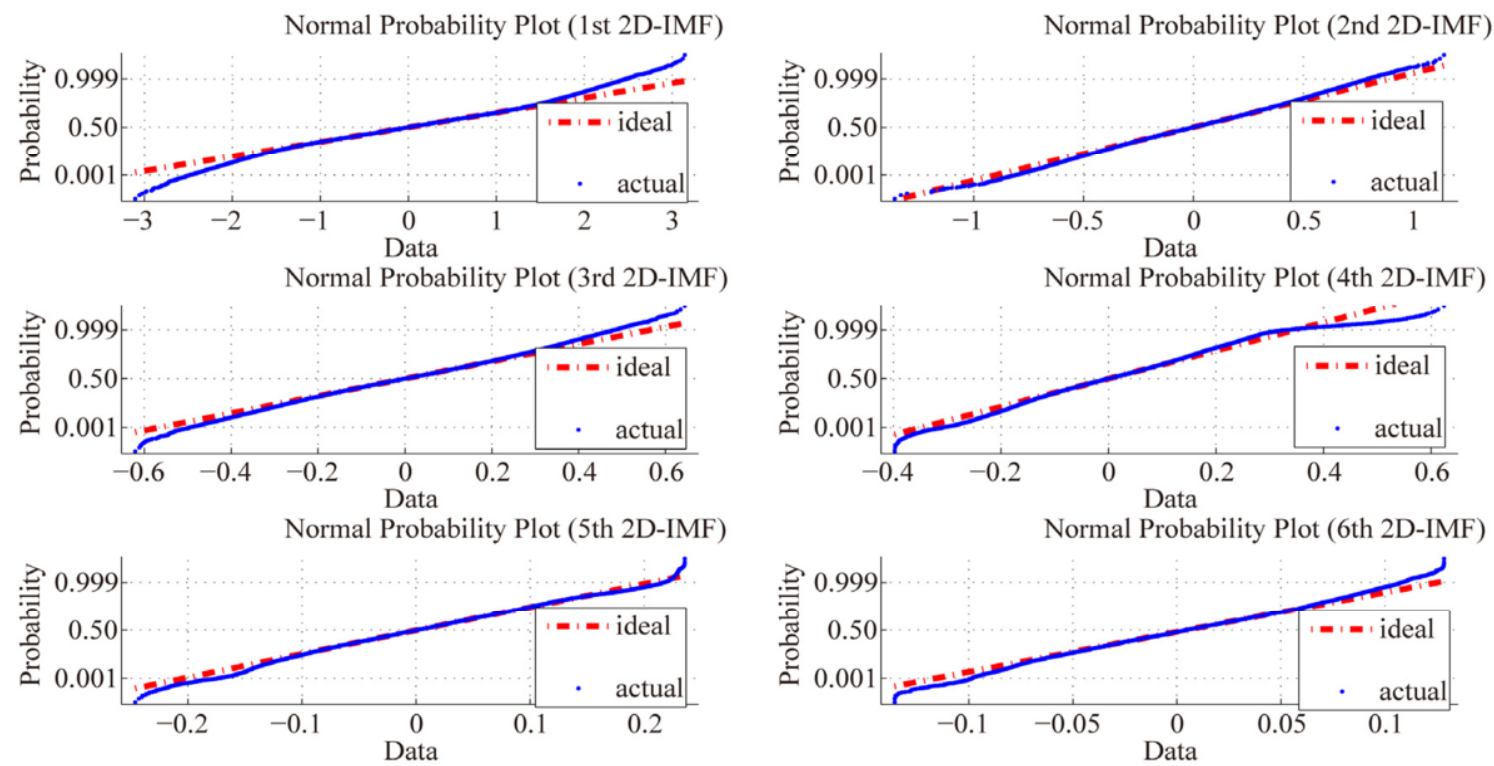

Fig. 1. Normal probability plot of each 2D-IMF of Gaussian white noise decomposed by BEMD. 
2D-IMF order from Figure 1. It implies that each 2D-IMF of the original image following the Gaussian distribution approximately follows the normal distribution.

Generally, the first 2D-IMF is mainly composed of the noise and a few details of the actual image. Therefore, the energy of the first 2D-IMF might be considered to be the first order noise energy roughly, which can be expresses as

$$
E_{1}=\sum_{i=1}^{N} \sum_{j=1}^{M} I_{1}^{2}(i, j)
$$

where the size of the decomposed image is $N \times M$. The energy of the $i$ th $\operatorname{IMF}(i=2,3, \ldots)$ decomposed by EMD can be estimated by [7]

$$
E_{i}=E_{1} \rho^{-i} / \beta
$$

where $\rho=2.01$ and $\beta=0.7190$ for the Gaussian white noise. The energy of the $i$ th $2 \mathrm{D}-\operatorname{IMF}(i=2,3, \ldots)$ of the Gaussian white noise image estimated by Eq. (3) is shown in Figure 2. Two pairs of $\rho$ and $\beta$ are used to estimate the energy with $\rho=2.0100, \beta=0.7190$ (green circle) and $\rho=2.0100, \beta=3.0000$ (blue star). It can be seen that the second pair of parameters better estimate the energy.

The unbiased estimation of the variance of the Gaussian distribution is given by

$$
\tilde{\sigma}_{i}^{2}=\frac{1}{N M-1} \sum_{i=1}^{N} \sum_{j=1}^{M} I_{i}^{2}(i, j)
$$

According to Eqs. (2)-(4), the Gaussian white noise standard deviation of the $i$ th $2 \mathrm{D}-\mathrm{IMF}(i=2,3, \ldots)$ of the image with the Gaussian white noise is

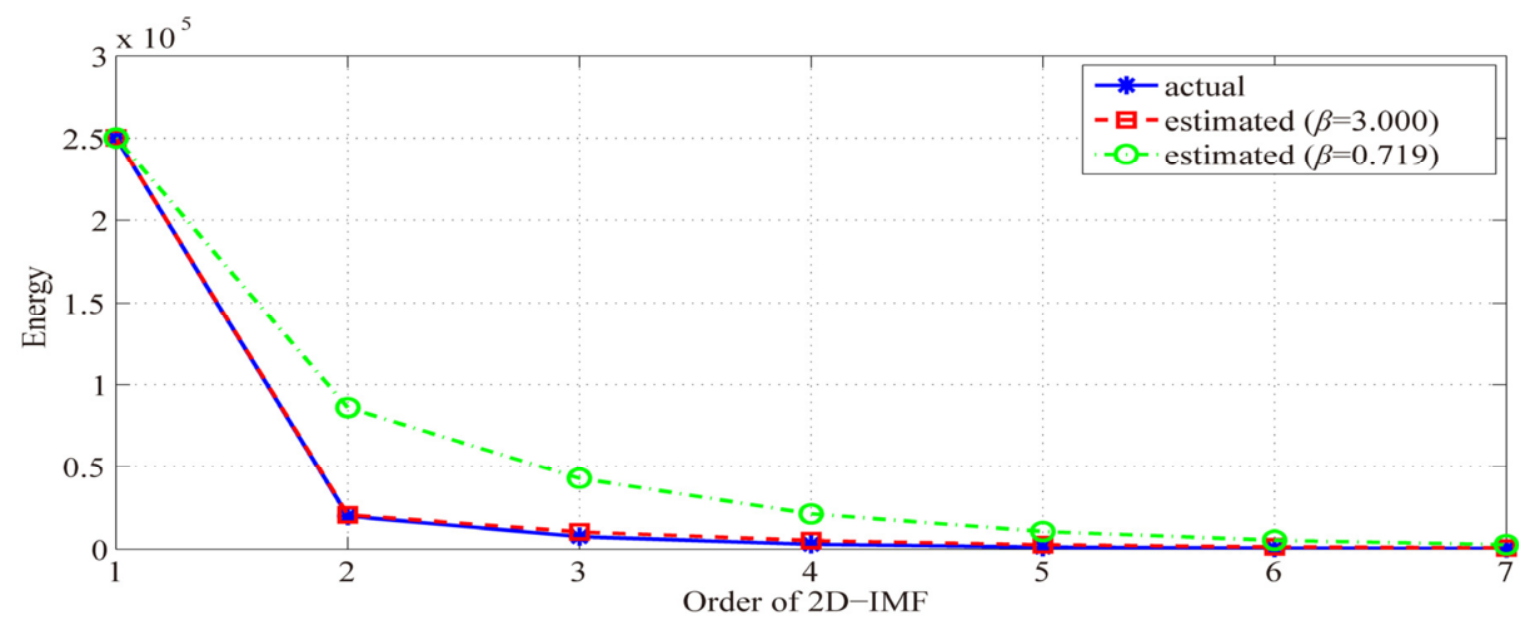

Fig. 2. Energy of each 2D-IMF of Gaussian white noise decomposed by BEMD. 


$$
\tilde{\sigma}_{i}=\tilde{\sigma}_{1} / \sqrt{\rho^{i} \beta}
$$

where $\tilde{\sigma}_{1}=\frac{1}{N M-1} \sum_{i=1}^{N} \sum_{j=1}^{M} I_{1}^{2}(i, j)$. As each 2D-IMF of the Gaussian white noise image follows the standard normal distribution, the probability of which the noise value of the $i$ th $(i=1,2,3, \ldots) 2 \mathrm{D}$-IMF belongs to the set $\left[-3 \tilde{\sigma}_{i}, 3 \tilde{\sigma}_{i}\right]$ is $99.73 \%$. If the pixel value of the $i$ th $(i=1,2,3, \ldots) 2 \mathrm{D}-\mathrm{IMF}$ is more than $3 \tilde{\sigma}_{i}$ or less than $-3 \tilde{\sigma}_{i}$, the pixel point is considered to include the actual image point. However, the self-adaptive threshold $T_{\mathrm{i}}$ of the $i$ th $(i=1,2,3, \ldots) 2 \mathrm{D}$-IMF should not be equal to $3 \tilde{\sigma}_{i}$, which is due to the fact that the first 2D-IMF includes a few details of the actual image. Therefore, in this work, the self-adaptive threshold $T_{i}$ is

$$
T_{i}=M \tilde{\sigma}_{i}
$$

where $M=2.8$.

The self-adaptive image denoising based on BEMD can be described as

1. Decompose the image with the Gaussian white noise using BEMD

2. Estimate $\tilde{\sigma}_{1}=\frac{1}{N M-1} \sum_{i=1}^{N} \sum_{j=1}^{M} I_{1}^{2}(i, j)$

3. Compute Ti $(\mathrm{i}=1,2,3, \ldots)$ by Eq. (6)

4. Perform the hard or the soft threshold denoising according to [16]

5. Perform $\tilde{I}=\sum_{i=1}^{S} \tilde{I}_{i}$ to obtain the image after denoising.

$$
\begin{aligned}
& \tilde{I}_{i}\left(Z_{i}^{m}\right)=\left\{\begin{array}{ll}
I_{i}\left(Z_{i}^{m}\right), & \max \left(\left|I_{i}\left(Z_{i}^{m}\right)\right|\right)>T_{i} \\
0, & \max \left(\left|I_{i}\left(Z_{i}^{m}\right)\right|\right) \leq T_{i}
\end{array}\right. \text { or } \\
& \tilde{I}_{i}\left(Z_{i}^{m}\right)=\left\{\begin{array}{lr}
I_{i}\left(Z_{i}^{m}\right) \frac{\max \left(\left|I_{i}\left(Z_{i}^{m}\right)\right|\right)-T_{i}}{\max \left(\left|I_{i}\left(Z_{i}^{m}\right)\right|\right)}, & \max \left(\left|I_{i}\left(Z_{i}^{m}\right)\right|\right)>T_{i} \\
0, & \max \left(\left|I_{i}\left(Z_{i}^{m}\right)\right|\right) \leq T_{i}
\end{array}\right.
\end{aligned}
$$

where $Z_{i}^{m}$ is the domain of which the periphery is the zero contour line. The $m$ th extrema of the $i$ th 2D-IMF belongs to domain $Z_{i}^{m}$. 
Table 1

Energy signal to noise ratio before and after denoising and time spent on denoising

\begin{tabular}{|c|c|c|c|}
\hline Method in [13] & Method in [17] & $\begin{array}{l}\text { Method based on wavelet analy- } \\
\text { sis }\end{array}$ & Presented method \\
\hline $\begin{array}{l}\text { Energy signal to noise } \text { ratio }_{22.5771} \\
\text { before denoising }(\mathrm{db})\end{array}$ & 22.5771 & 22.5771 & 22.5771 \\
\hline $\begin{array}{l}\text { Energy signal to noise ratio } \\
\text { after denoising }(\mathrm{db})\end{array}$ & 37.1706 & 41.4516 & 43.5262 \\
\hline Time spent on denoising (hour) 2.03 & 2.27 & 0.08 & 0.09 \\
\hline
\end{tabular}

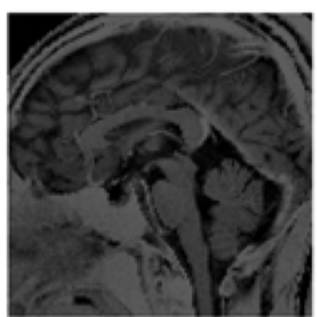

(a) Original MRI

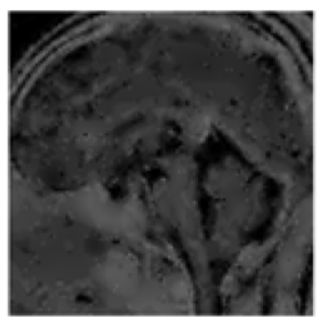

(d) Denoised MRI using method in [17]

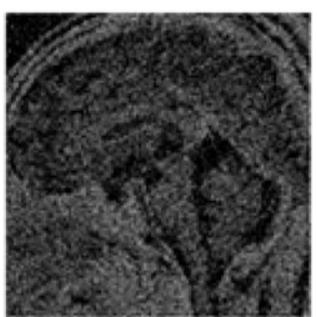

(b) MRI with Gauss white noise

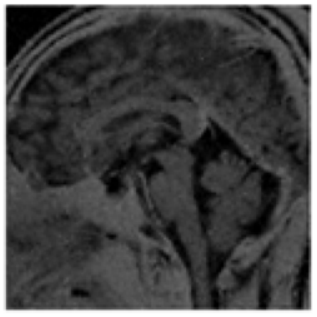

(e) Denoised MRI using method based on wavelet analysis

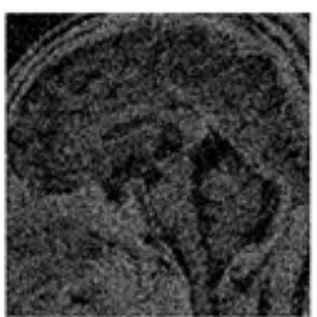

(c) Denoised MRI using method in [13]

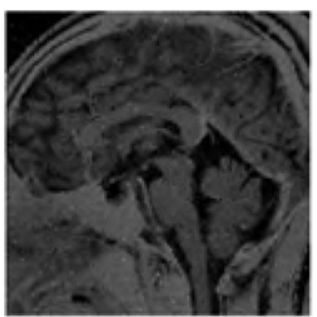

(f) Denoised MRI using presented method

Fig. 3. MRIs of the brain before and after denoising using the methods based on EMD and BEMD.

\section{Results and discussion}

The self-adaptive image denoising method based on BEMD should be applied to the magnetic resonance image (MRI) denoising of the brain. The original and the Gaussian white noise MRIs of the brain are shown in Figures 3(a) and 3(b), respectively. The images after denoising by the method in [13], the self-adaptive image denoising method based on EMD in [17], the method based on discrete wavelet analysis and the presented method are shown in Figures 3(c)-3(f), respectively. The energy signal to noise ratios before and after denoising and the time spent on denoising are shown in Table 1. It can be seen that the MRI after denoising by the presented method is the most distinctive. Moreover, the presented method produces higher signal to noise ratio after image denoising and costs less time compared with the method presented in [13] and [17]. 


\section{Conclusion}

The self-adaptive image denoising method based on BEMD was presented in this work. It does not require any human intervention and can be solely achieved using the input image. The proposed method was applied in MRI denoising of the brain, which shows that the presented method has a better denoising performance and takes less time compared with methods based on EMD [13] and [17].

\section{Acknowledgement}

The work is supported by science and technology research project of education department of Liaoning province of China (Grant No. L2011092), 2012 science and technology plan of ministry of housing and urban-rural development of China (Grant No. 2012-K8-29), science and technology foundation of Liaoning province of China (Grant No. 2013020012) and China ministry of education new century excellent person support plan (Grant No. NCET-12-0105).

\section{References}

[1] R.M. Yan, L. Shao and Y. Liu, Nonlocal hierarchical dictionary learning using wavelets for image denoising, IEEE Transactions on Image Processing 22 (2013), 4689-4698.

[2] S.G. Chang, B. Yu and M. Vetterli, Adaptive wavelet thresholding for image denoising and compression, IEEE Transactions on Image Processing 9 (2000), 1532-1546.

[3] F. Wang and Z. Ji, Application of the dual-tree complex wavelet transform in biomedical signal denoising, Bio-Medical Materials and Engineering 24 (2014), 109-115.

[4] J. Portilla, V. Strela, M.J. Wainwright and E.P. Simoncelli, Image denoising using scale mixtures of gaussians in the wavelet domain, IEEE Transactions on Image Processing 12 (2003), 1338-1351.

[5] S. Vaisman, S.Y. Salem, G. Holcberg and A.B. Geva, Passive fetal monitoring by adaptive wavelet denoising method, Computers in Biology and Medicine 42 (2012), 171-179.

[6] N.E. Huang, Z. Shen, S.R. Long, M. Wu, H. Shih et al., The empirical mode decomposition and the Hilbert spectrum for non-linear and non-stationary time series analysis, Proceedings of the Royal Society of London Series AMathematical Physical and Engineering Sciences 454 (1998), 903-995.

[7] N.E. Huang and S.S.P. Shen, Statistical significance test of Intrinsic Mode Functions, in: Hilbert-Huang transform and its applications, World Scientific, Singapore, 2005. pp. 107-125.

[8] P. Flandrin, G. Rilling and P. Gonçalvés, Empirical mode decomposition as a filter bank, IEEE Signal Processing Letters 11 (2004), 112-114.

[9] Z.H. Wu and N.E. Huang, A study of the characteristics of white noise using the empirical mode decomposition method, Proceedings of the Royal Society A- Mathematical Physical and Engineering Sciences 460 (2004), 1597-1611.

[10] Z.Q. Sun, J.M. Zhou and P. Zhou, Application of Hilbert-Huang transform to denoising in vortex flowmeter, Journal of Central South University of Technology 13 (2006), 501-504.

[11] J.T. Tang, Q. Zou, Y. Tang, B. Liu and X.K. Zhang, Hilbert-Huang transform for ECG de-noising, 1st International Conference on Bioinformatics and Biomedical Engineering, 2007, 664-667.

[12] L. Qian, G.P. Xu, W.F. Tian and J.P. Wang, A novel hybrid EMD-based drift denoising method for a dynamically tuned gyroscope (DTG), Measurement 42 (2009), 927-932.

[13] X.L. Hu and M. Jin, Improved algorithm of image signal denoising based on EMD, Electronic Measurement Technology 32 (2009), 58-61.

[14] Y. Yan and Z.Z. Cui, Noise and zero excursion elimination of electrostatic detection signals based on EMD and wavelet transform, 2nd International Congress on Image and Signal Processing, 2009, 4794-4798.

[15] V. Agarwal and L.H. Tsoukalas, Denoising electrical signal via empirical mode decomposition, 2007 iREP Symposium- Bulk Power System Dynamics and Control - VII, Revitalizing Operational Reliability, 2007, 72-77.

[16] Y. Kopsinis and S. McLaughlin, Development of EMD-based denoising methods inspired by wavelet thresholding, IEEE Transactions on Signal Processing 57 (2009), 1351-1362. 
[17] S. Guo, G.C. Gu, C.Y Li, F.J. Luan and X.Y. Song, Adaptive image denoising based on EMD, Computer Engineering and Applications 49 (2013), 12-16

[18] A. Linderhed, Variable sampling of the empirical mode decomposition of two-dimensional signals, International Journal of Wavelets, Multiresolution and Information Processing 3 (2005), 435-452.

[19] J.C. Nunes, Y. Bouaoune, E. Delechelle, O. Niang and P. Bunel, Image analysis by bidimensional empirical mode decomposition, Image and Vision Computing 21 (2003), 1019-1026.

[20] J.C. Nunes, S. Guyot and E. Deléchelle, Texture analysis based on local analysis of the bidimensional empirical mode decomposition, Machine Vision and Applications 16 (2005), 177-188.

[21] M.A.S. Bhuiyan, R.R. Adhami and J.F. Khan, Fast and adaptive bidimensional empirical mode decomposition using order-statistics filter based envelope estimation, EURASIP Journal on Advances in Signal Processing, 2008, 728356.

[22] Y. Pei, Y.G. Wu and D.C. Jia, Image denoising based on bidimensional empirical mode decomposition, 2011 International Conference on Mechatronic Science, Electric Engineering and Computer, 2011, 1122-1125.

[23] S.M.A. Bhuiyan1, R.R. Adhami, H.S. Ranganath and J.F. Khan, Aurora image denoising with a modified bidimensional empirical mode decomposition method, IEEE Southeastcon, 2008, 527-532. 